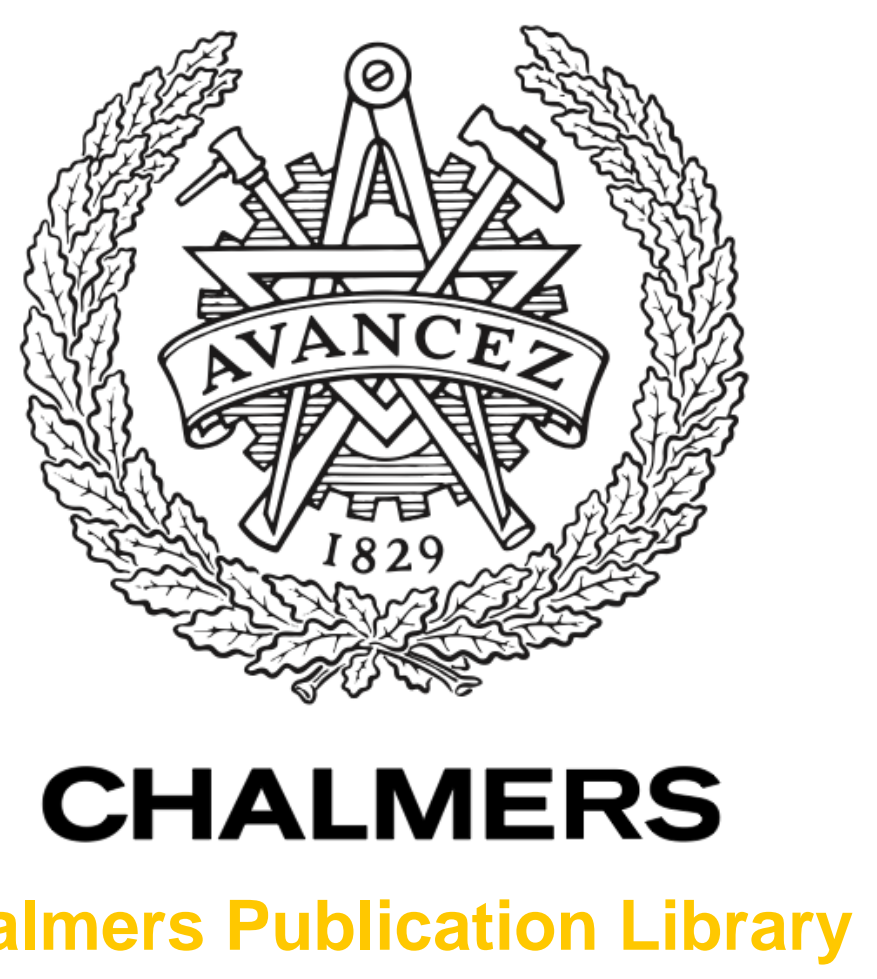

Chalmers Publication Library

\title{
Code Rate Optimization in Elastic Optical Networks
}

This document has been downloaded from Chalmers Publication Library (CPL). It is the author's version of a work that was accepted for publication in:

\section{European conference on optical communications}

Citation for the published paper:

Zhao, J. ; Yan, L. ; Agrell, E. et al. (2015) "Code Rate Optimization in Elastic Optical

Networks". European conference on optical communications

Downloaded from: http://publications.lib.chalmers.se/publication/223990

Notice: Changes introduced as a result of publishing processes such as copy-editing and formatting may not be reflected in this document. For a definitive version of this work, please refer to the published source. Please note that access to the published version might require a subscription. 


\title{
Code Rate Optimization in Elastic Optical Networks
}

\author{
Juzi Zhao, Li Yan, Henk Wymeersch, and Erik Agrell
}

Chalmers University of Technology, Gothenburg, Sweden, juzi@chalmers.se

\begin{abstract}
We combine adaptive coding with nonlinear impairment-aware routing and spectrum allocation problem in elastic optical networks. An integer linear program formulation and low-complexity heuristics are proposed. Results indicate that over $50 \%$ bandwidth reduction can be obtained due to code-rate optimization.
\end{abstract}

\section{Introduction}

Elastic optical networks are an ideal candidate for core backbone networks traffic demands increase, because of their ability to efficiently utilize the fiber bandwidth. Optical orthogonal frequency division multiplexing (OOFDM) is one of the technologies to enable elastic optical networks, where the fiber bandwidth is divided into multiple finegranularity subcarriers ${ }^{1}$.

Although the routing and spectrum allocation (RSA) problem for optical networks has been studied for over one decade, resource allocation with adaptive coding is a recently emerging research topic. The routing, code, and wavelength assignment problem for WDM fixed grid optical networks has been addressed ${ }^{2-4}$. The RSA problem with adaptive code rates has been studied for transparent elastic networks with dynamic traffic ${ }^{5}$ and for translucent and opaque elastic networks with static trafic ${ }^{6,7}$. However, the combination of transparent networks and static traffic has not been considered yet.

In this paper, the RSA problem together with code rate optimization, assuming static connection requests in transparent OOFDM-based elastic optical networks, is studied for the first time, accounting for nonlinear physical impairments of each connection.

\section{Model and Problem Statement}

We adopt a physical layer model ${ }^{8}$ to estimate the impairments of each connection. The signal-tonoise ratio (SNR) for connection $i$ using route $r_{i}$ can be calculated as $\mathrm{SNR}_{i}=G /\left(G_{\mathrm{ASE}}+G_{\mathrm{NLI}}\right)$, where $G$ is the signal power spectral density (PSD). The PSD of the amplified spontaneous emission (ASE) noise is $G_{\mathrm{ASE}}=\sum_{l \in r_{i}} N_{l} G_{\mathrm{ASE}}^{0}$, where $G_{\text {ASE }}^{0}$ is the constant PSD in a single span, and $N_{l}$ is the number of spans on link $l$. The PSD of the nonlinear impairments (NLI) is $G_{\mathrm{NLI}}=$ $\sum_{l \in r_{i}} N_{l} G_{\mathrm{NLI}}^{0}$, where $G_{\mathrm{NLI}}^{0}$ is the PSD of the NLI noise in a single span, which is a nonlinear func-

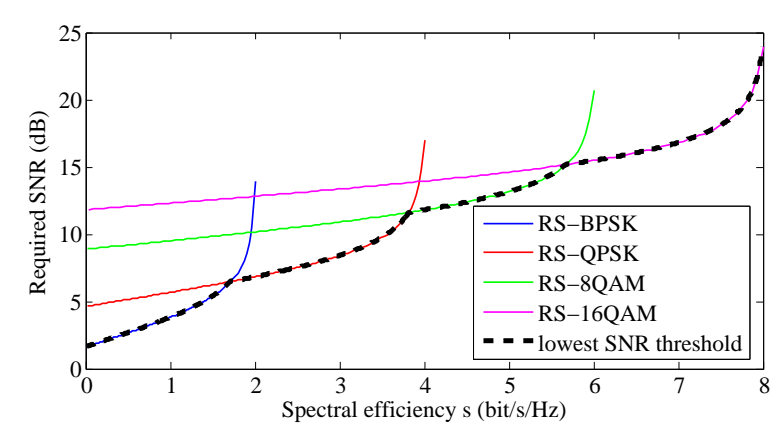

Fig. 1: RS code with $n=255$; spectrum efficiency $s$ v.s. symbol SNR threshold

tion of connection's bandwidth and center frequency spacing between connections.

The family of Reed-Solomon codes $\operatorname{RS}(n, k)$ is adopted since it is the most common code family in commercial optical networks and the same RS decoder hardware can operate with different code rates. With $n$-byte codewords, $k$ information bytes, and a modulation format that transmits $q$ bit/symbol, the spectral efficiency in information bits per symbol is $s=2 k q / n$. For a particular bit rate, the number of subcarriers depends on $s$. Given a post-forward error correction (postFEC) bit error rate (BER) requirement, the required pre-FEC symbol SNR can be calculated for each combination of modulation format and code rate as shown in Fig. $1^{9,10}$. For each value of the spectral efficiency $s$, there is one modulation format that requires the lowest symbol SNR threshold, which is highlighted by the dashed curve.

The network is represented by a graph $G(V, E)$ with node set $V$ and link set $E$. There are two fibers with opposite directions on each link $l \in E$. There are $S$ subcarriers on each fiber, each with bandwidth $C \mathrm{GHz}$. $M$ modulation formats can be used for each subcarrier. A Reed-Solomon code $\mathrm{RS}(n, k)$ with flexible $k$ and a fixed $n=255$ are allocated to each connection. The off-line traffic demand is represented by an all-to-all symmetric matrix. For each connection, we need to assign a route, a modulation format, a code rate, and a contiguous subcarrier band in order to satisfy its 
bit rate requirement and SNR requirements. The goal is to minimize the maximum bandwidth in the network.

\section{ILP Formulation}

The problem can be formulated and solved as an integer linear programming (ILP) problem. Due to the space limit, we only present modifications to our previous work ${ }^{11}$, where the routing, modulation format and spectrum allocation problem with the same nonlinear physical layer impairment model was studied, without code rate allocation.

As shown in Fig. 1, the minimal required SNR is a nonlinear function of the spectral efficiency $s$. In order to include it in the ILP formulation, we lower-bound its inverse $N_{S R}{ }^{\text {req }}=1 /$ SNR $^{\text {req }}$ by a piecewise linear function of $T=1 / s$ : we divide the range of $T$ into multiple segments, and use a linear function $y=a_{g} x+b_{g}$ to approximate the NSR of each segment $g$ with segment $\left[T^{g}, T^{g+1}\right]$; however, according to $G \mathrm{NSR}{ }^{\text {req }} \geq G_{\mathrm{ASE}}+G_{\mathrm{NLI}}$, there is a constraint that the approximated value of $N S R^{\text {req }}$ should be no greater than the actual value (underestimation or lower bound) as shown in Fig. 2. The segments are found through an optimization procedure.

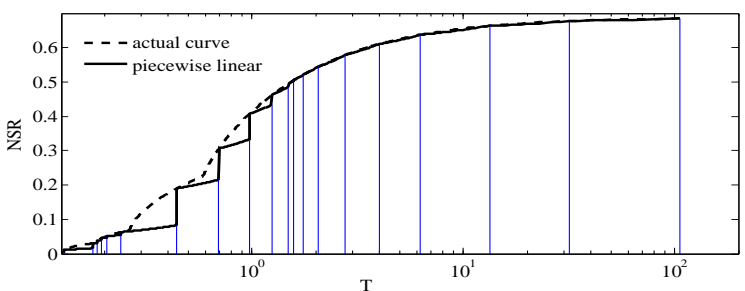

Fig. 2: Piecewise linear function $T$ v.s. NSR.

The ILP problem ${ }^{11}$ comprises the following variables: $B_{i} \in \mathbb{N}$, the number of subcarriers allocated to connection $i$; $p_{i l} \in \mathbb{B}$, which is 1 if link $l$ is on the route assigned to connection $i$; and $t_{i l} \in \mathbb{R}_{\geq 0}$, the value of $G_{\mathrm{NLI}}^{l}$ of connection $i$ on link $l ; T_{i} \in \mathbb{R}_{\geq 0}$, the value of $T=1 / s$ allocated to connection $i$; $d_{i g} \in \mathbb{B}$, which is 1 if $T_{i}$ is in the segment $\left[T^{g}, T^{g+1}\right]$; $\mathrm{NSR}_{i}^{\text {req }} \in \mathbb{R}_{>0}$, one over the required SNR threshold of connection $i$ with the allocated spectral efficiency; and $m_{i k} \in \mathbb{B}$, which is 1 if $k$ subcarriers are allocated to connection $i$.

These allow us to formulate additional constraints into ${ }^{11}$ as follows:

$$
\begin{aligned}
& B_{i}=T_{i} \Lambda_{i} \quad \forall i \\
& \sum_{k} k m_{i k}=B_{i} \quad \forall i \\
& G \mathrm{NSR}_{i}^{\text {req }} \geq G_{\mathrm{ASE}}^{0} \sum_{l} p_{i l} N_{l}+\sum_{l} N_{l} t_{i l} \quad \forall i \\
& \sum_{g} d_{i g}=1 \quad \forall i \\
& T_{i} \geq \theta\left(d_{i g}-1\right)+T^{g} \\
& T_{i} \leq \theta\left(1-d_{i g}\right)+T^{g+1} \\
& \mathrm{NSR}_{i}^{\text {req }} \leq \theta\left(1-d_{i g}\right)+a_{g} T_{i}+b_{g} \\
& \mathrm{NSR}_{i}^{\text {req }} \geq \theta\left(d_{i g}\right)+a_{g} T_{i}+b_{g}
\end{aligned}
$$

where $\Lambda_{i}$ (the bit rate requirement of connection $i$ ) and $G$ are input parameters; $\theta$ denotes a large number; constraint (a) relates the allocated bandwidth to the assigned spectral efficiency; (b) relates $A_{i m}$ to $B_{i}$; (c) ensures that the SNR requirement of each connection is satisfied; (d)-(h) ensure that $T_{i}$ belongs to only one segment and relate the allocated spectral efficiency to the required SNR threshold.

\section{Proposed Heuristics}

Although the routing, code, and spectrum assignment of all connections can be optimally solved by the ILP, it is very time consuming due to the involved integer constraints, thus it is only suitable for small networks with a few connection requests. For larger networks, we propose two heuristics: group ILP (GILP) and connection list $(\mathrm{CL})$ by extending our previous work ${ }^{11}$. Due to the space limit, only the main idea and modifications of each algorithm are mentioned as below.

The GILP groups the connections in successive nonoverlapping groups of size $\eta \geq 1$, and it calls the ILP for each group taking the previously allocated connections into account. In addition, an adaptive SNR margin is used to estimate nonlinear interference from future calls.

The CL heuristic allocates resources to the set of connections one by one. For each particular value of the spectral efficiency (which may be associated with multiple combinations of modulation format and code rate), an SNR threshold is precomputed (the minimum one is selected if there are multiple values) and kept in a look-up table. For each connection, CL considers the possible combination of spectral efficiency $s$ and starting subcarrier index for allocation, and assigns a cost to every link based on the impairment effect, and then it finds the minimum cost path by utilizing the Dijkstra shortest path algorithm. An SNR margin for future connections is included.

\section{Numerical Results}

The simulation results for two network topologies $^{12}$ are presented: a small 6-node network and the larger 14-node Deutsche Telekom (DT) network. The same network parameters are set as in ${ }^{11}$. Each connection's post-FEC BER requirement is $10^{-12}$. Four modulation formats are used: BPSK, QPSK, 8-QAM. The bit rate requirement of each connection is uniformly distributed from 0 to 312.5 Gbps. For each connection, an $\mathrm{RS}(255, k)$ code is used, with $k$ optimized jointly with the RSA. The results are compared with RSA results using the standard $\mathrm{RS}(255,239)$ code for 


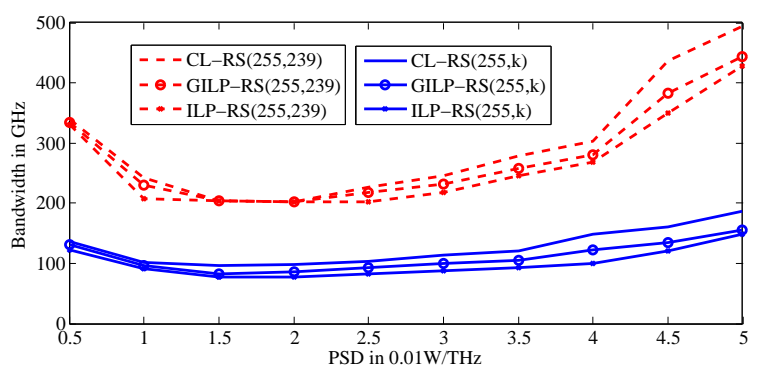

Fig. 3: Bandwidth v.s. PSD in 6-node network

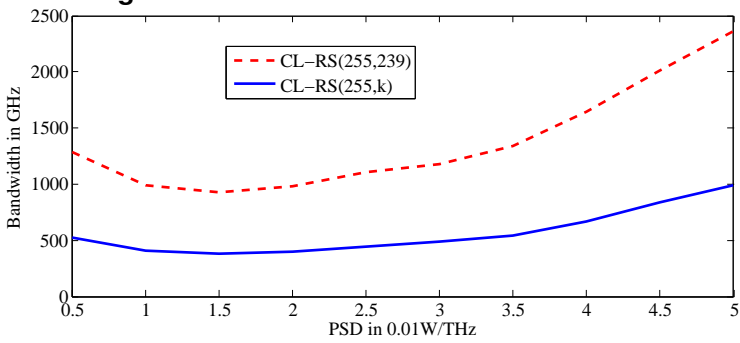

Fig. 4: Bandwidth v.s. PSD in 14-node network

all connections. The resulting bandwidths as a function of the PSD are shown in Figs. 3 and 4, averaged over ten connection request matrices. It is indicated that code rate optimization can save $51.7 \%$ and $59.5 \%$ bandwidth when PSD is 0.02 $\mathrm{W} / \mathrm{THz}$ in the small and large network, respectively. Also, the optimal bandwidth is less sensitive to the choice of PSD when the code rate is optimized.

The ranges of the allocated code rates as functions of PSD and link-length stretching factor (when PSD is $0.02 \mathrm{~W} / \mathrm{THz}$ ) in the 6-node network are shown in Figs. 5 and 6, respectively. When RS code rates are optimized together with the RSA, we observe that a wide variety of code rates is used (ranging from 0.35 to 0.95 ), with an average of around 0.68 . The average overhead is thus $41 \%$, which is much larger than $7 \%$ user in standards ${ }^{10}$. From Fig. 6, we also see that when links are longer and nonlinearities become more prominent, lower code rates should be used.

\section{Conclusions}

By including realistic code rate assignment, novel routing and spectrum allocation algorithms were proposed for elastic networks based on OOFDM technology. Simulations show that the proposed algorithms save over $50 \%$ in bandwidth compared to algorithms with fixed code rate assignment.

\section{Acknowledgements}

The research was funded by the Swedish Research Council (VR) under grant no. 2012-5280.

\section{References}

[1] I.B. Djordjevic and B. Vasic, "Orthogonal Frequency Division Multiplexing for High-Speed Optical Transmission,"

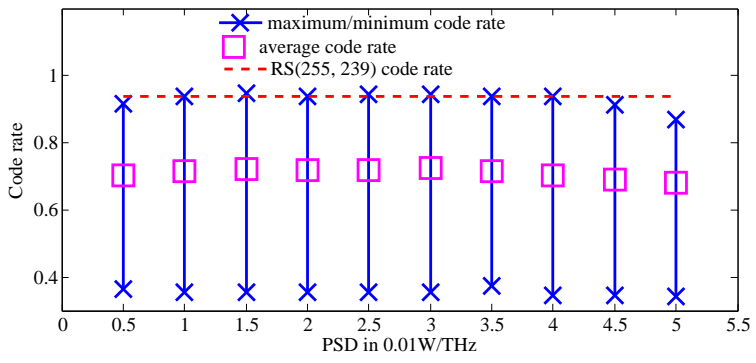

Fig. 5: Code rate v.s. PSD in 6-node network

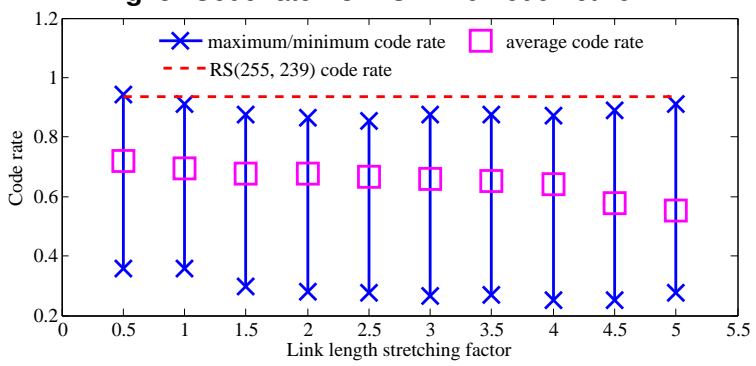

Fig. 6: Code rate v.s. link length stretching factor in 6-node network

Opt. Expr., Vol. 14, no. 9, p. 3767 (2006).

[2] D.A.A. Mello et al., "Optical Networking With VariableCode-Rate Transceiver," J. Lightwave Technol., Vol. 32, no. 2, p. 257 (2014).

[3] D. J. Ives et al., "Assessment of Options for Utilizing SNR Margin to Increase Network Data Throughput," Proc. OFC, M2I.3, Los Angeles (2015).

[4] H.D. Thanh et al., "On the Spectrum-efficiency of Transparent Optical Transport Network Design with Variablerate Forward Error Correction Codes," Proc. ICACT, Pyeongchang (2014).

[5] N. Sambo et al., "Routing, Code, and Spectrum Assignment (RCSA) in Elastic Optical Networks," Proc. OFC, W1I.1, Los Angeles (2015).

[6] H. Khodakarami et al., "Flexible Optical Networks: An Energy Efficiency Perspective," J. Lightwave Technol., Vol. 32, no. 21, p. 3958 (2014).

[7] F. I. Cerutti et al., "Trading Regeneration and Spectrum Utilization in Code-Rate Adaptive Flexi-Grid Networks," J. Lightwave Technol., Vol. 32, no. 23, p. 4496 (2014).

[8] P. Johannisson and E. Agrell, "Modeling of Nonlinear Signal Distortion in Fiber-Optic Networks," J. Lightwave Technol., Vol. 32, no. 23, p. 4544 (2014).

[9] K. Szczerba et al., "Comparison of Intersymbol Interference Power Penalties for OOK and 4-PAM in Short-range Optical Links," J. Lightwave Technol., Vol. 31, no. 22, p. 3525 (2013).

[10] G.975 : Forward error correction for submarine systems, ITU-T Std, 2000.

[11] J. Zhao et al., "Nonlinear Impairment Aware Resource Allocation in Elastic Optical Networks," Proc. OFC, M2I.1, Los Angeles (2015).

[12] K. Christodoulopoulos et al., "Elastic Bandwidth Allocation in Flexible OFDM-Based Optical Networks," J. Lightwave Technol., Vol. 29, no. 9, p. 1354 (2011). 